\title{
What Should an Ideal Adult Spinal Deformity Classification System Consist of ?: Review of the Factors Affecting Outcomes of Adult Spinal Deformity Management
}

\author{
J. Naresh-Babu ${ }^{1}$, Arun-Kumar Viswanadha ${ }^{1}$, Manabu Ito ${ }^{2}$, Jong-Beom Park ${ }^{3}$ \\ ${ }^{1}$ Department of Spine Surgery, Mallika Spine Centre, Guntur, India \\ ${ }^{2}$ Department of Spine and Spinal Cord Disorders, National Hospital Organization, Hokkaido Medical Center, Sapporo, Japan \\ ${ }^{3}$ Department of Orthopaedic Surgery, Uijeongbu St. Mary's Hospital, College of Medicine, The Catholic University of Korea, Uijeongbu, Korea
}

\begin{abstract}
This literature review aims to determine potential clinical factors or comorbidities besides radiological parameters that affect the outcome of adult spinal deformity (ASD) management and review existing classifications associated with ASD. ASD is a multifactorial disease that comprises pathologies like radiological spine deformity, coexistence of spinal canal stenosis, radiculopathy, and multiple comorbidities. The available classification systems of ASD are predominantly based on radiological parameters and do not consider related clinical conditions. ASD patients with different combinations of these parameters behave differently and need different management strategies. We conducted a narrative literature review with search limited to English language of PubMed/MEDLINE using Medical Subject Heading (MeSH) terms. The terms specific to the review were ASD and several other related terminologies. We analyzed the information of the selected papers including factors affecting surgical outcomes for degenerative scoliosis. We reviewed 614 citations. Based on the inclusion criteria, 39 citations were selected for full-text retrieval; of these, 28 were excluded because of not fulfilling the inclusion criteria. Thus, 11 studies were selected and included for the final analysis. The presence of leg pain, spinal stenosis, obesity, osteoporosis, smoking, and age of patients were major influencing factors. Furthermore, the factors included in the available classifications, such as the Scoliosis Research Society-Schwab classifications, were reviewed and results were tabulated. This review highlights the significance of neurological symptoms, spinal stenosis, osteoporosis, obesity, age, and smoking, which markedly affect the management of ASD. With increasing number of patients being diagnosed and treated with ASD, there has been a growing need to comprehensively classify these patients into clinicoradiological subgroups.
\end{abstract}

Keywords: Thoracolumbar spine; Adult spinal deformity; Classification; Narrative review; Factors influencing outcomes

\section{Introduction}

Adult de novo scoliosis implies the development of scoliotic curves after skeletal maturity, without any history of deformity. Reportedly, it occurs most commonly in later stages of life and is related to back pain, radicular pain, and, most importantly, complicated surgical outcomes partially associated with comorbidities in older age [1].

Received Dec 2, 2018; Revised Feb 21, 2019; Accepted Mar 10, 2019

Corresponding author: J. Naresh-Babu

Department of Spine Surgery, Mallika Spine Centre, 12-12-30, Old Club Road, Kothapet, Guntur, Andhra Pradesh 522001, India

Tel: +91-9989426898, E-mail: nareshspine@yahoo.com 
The process of aging affects all structures associated with the spine, such as the vertebral body, ligaments, intervertebral discs, zygapophyseal joints, and muscles, which exerts a domino-like effect resulting in the compression/wedging of vertebral bodies, decline in the intervertebral disc height, decreased lumbar lordosis, rotation, and translation of vertebra. The final result is neural compression caused by ligamentum flavum hypertrophy, facet hypertrophy, disk degeneration resulting in prolapse, and subluxation of the vertebra.

The management of patients with adolescent idiopathic scoliosis is entirely driven by cosmesis and radiological deformity, whereas the spectrum of adult spinal deformity (ASD) besides a radiological deformity contains multiple facets like low back pain, nerve root compression resulting in radiculopathy or neurological claudication, osteoporosis, functional disability, and other comorbidities eventually affecting the activities of daily living and quality of life. Patients with different combinations of these parameters behave differently and require different management strategies (Fig. 1). All available classifications of ASD are predominantly based on radiological parameters and do not consider such related conditions; classifying these patients basing on pure radiological parameters is not sufficient.

Hence, this study aims to review existing classifications addressing ASD and identify clinical factors or comorbidities affecting the outcome in ASD.

\section{Methodology}

\section{Study design}

This study is literature review.

\section{Search}

We conducted a literature search limited to English
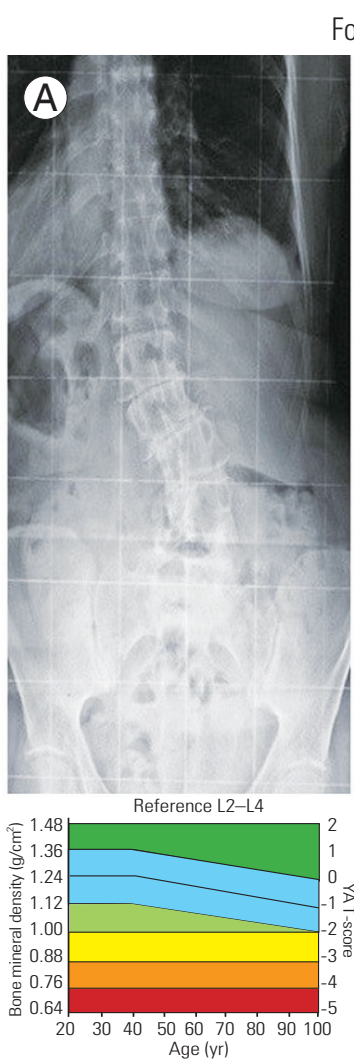

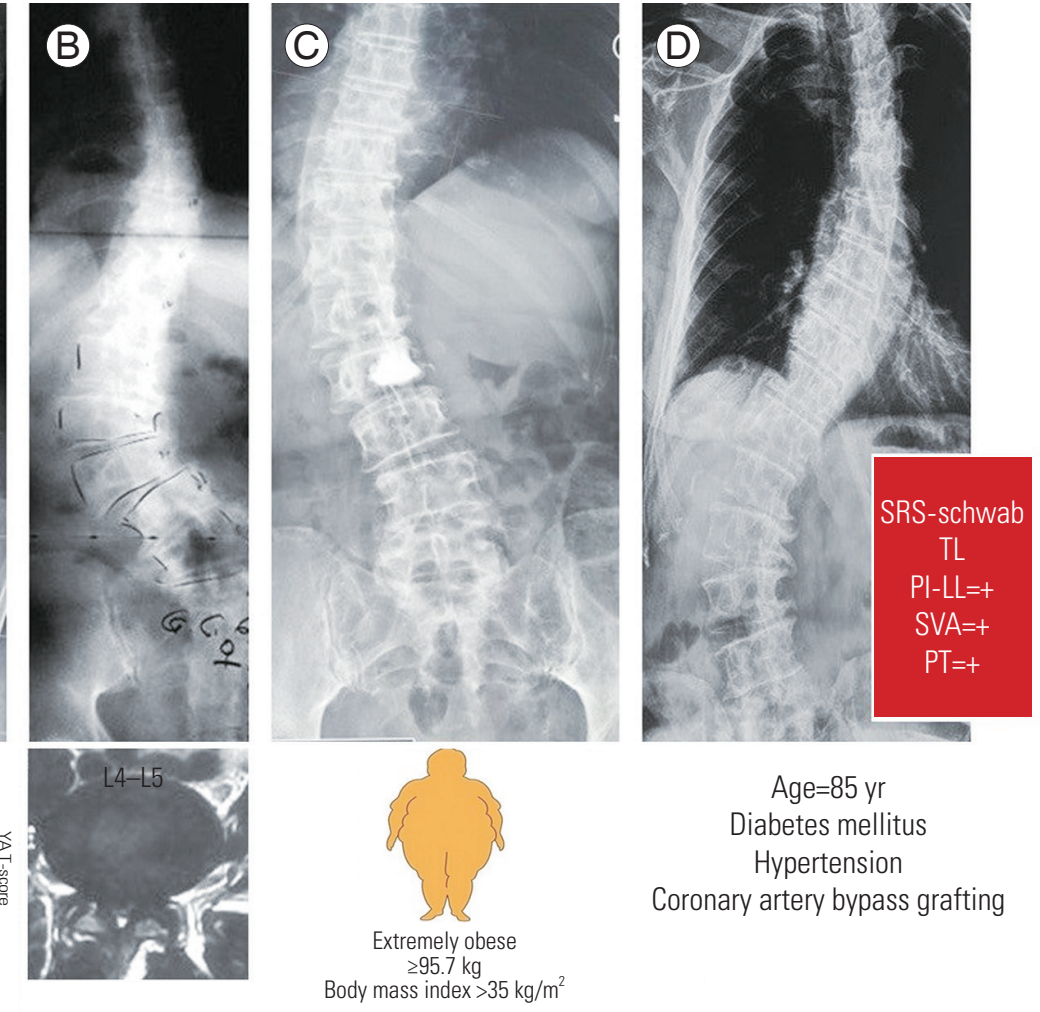

Four radiologically identical adult spinal deformity patients

Fig. 1. According to the SRS-Schwab classification of adult scoliosis, all four patients belong to the same category. However, $(\mathbf{A})$ is associated with severe osteoporosis where the risk of implant failures is high, $(\mathbf{B})$ is associated with lumbar canal stenosis where the major concern is neurological claudication, $(C)$ is associated with severe obesity that increases the risk of anesthesia, and (D) is associated with multiple comorbidities that increase the risk of surgery as a whole. SRS, Scoliosis Research Society; TL, thoracolumbar kyphosis; PI, pelvic incidence; LL, lumbar lordosis; SVA, sagittal vertical axis; PT, pelvic tilt. 
language of PubMed/MEDLINE using Medical Subject Heading (MeSH) terms. The terms specific to the review were 'degenerative scoliosis' OR 'adult spinal deformity' OR 'adult degenerative scoliosis' OR 'adult deformity' these were combined with 'factors influencing' OR 'factors affecting' OR 'parameters affecting' OR 'parameters influencing' OR 'clinical impact' OR 'comorbidities' OR 'clinical outcomes.'

\section{Date searched}

The data were searched from 1950 to October 2018.

\section{Inclusion criteria}

Studies were included when the factors affecting surgical outcomes for degenerative scoliosis were detailed.

\section{Exclusion criteria}

Studies excluded were case reports, deformity because of infection/trauma/tumor, case series with $<10$ patients/ group, animal, in vitro, and biomechanical studies.

\section{Analysis}

We collected descriptive statistics, means, standard deviations, and ranges from original articles. The collected data were not pooled together because of the heterogeneity of studies.

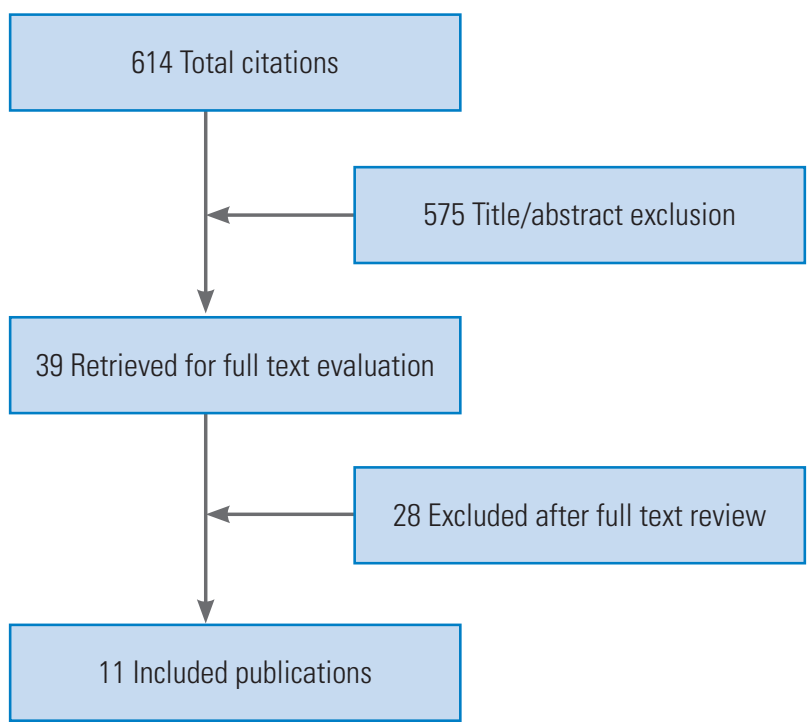

Fig. 2. The flowchart showing the results of the literature search.

\section{Results}

We reviewed 614 citations in this study. After title and abstract exclusion, 39 citations were selected for full-text retrieval; of these, 28 were excluded because of not fulfilling the inclusion criteria. Thus, 11 studies were selected and included for the final analysis (Fig. 2).

Publications included in this study for review are as follows (Table 1): (1) Yagi et al. [2] in a retrospective study validated the prevalence of low bone mineral density (BMD) in female patients with ASD; (2) Xu et al. [3] investigated the prevalence of degenerative scoliosis in Chinese Han population along with its correlation with age, gender, BMD, and body mass index (BMI); (3) Fu et al. [4] in a retrospective cohort study analyzed the correlation between the BMI and surgical outcomes in patients with ASD; (4) Urrutia et al. [5] in a cross-sectional study demonstrated the correlation of BMD, age, and BMI with lumbar degenerative scoliosis in post-menopausal females; (5) Soroceanu et al. [6] in a retrospective review determined the risk factors for medical complications in patients with ASD undergoing surgery; (6) Kebaish et al. [7] in a retrospective study analyzed the correlation between adult scoliosis and age, race, and gender; (7) Wang et al. [8] in a prospective study classified the associated lumbar canal stenosis (LCS) in ASD and suggested the treatment options accordingly; (8) Smith et al. [9] in a secondary analysis of prospectively collected data from the Spinal Deformity Study Group identified clinical parameters that distinguish between best and worst outcomes following adult deformity correction; (9) Adogwa et al. [10] in a retrospective study investigated the correlation between the preoperative serum albumin level and complication rates following spine fusion in adult degenerative and deformity patients; (10) Worley et al. [11] collected the nationwide inpatient sample database and analyzed predictors of morbidity and mortality in ASD surgery; and (11) Acosta et al. [12] in a retrospective study identified the factors predictive of perioperative complications for ASD surgery.

\section{Comparison of Data in Included Studies}

\section{Prevalence}

The incidence of adult deformity was $8.85 \%$ in Kebaish et al. [7], $13.27 \%$ in Xu et al. [3], and $12.9 \%$ in Urrutia et al. [5]. 


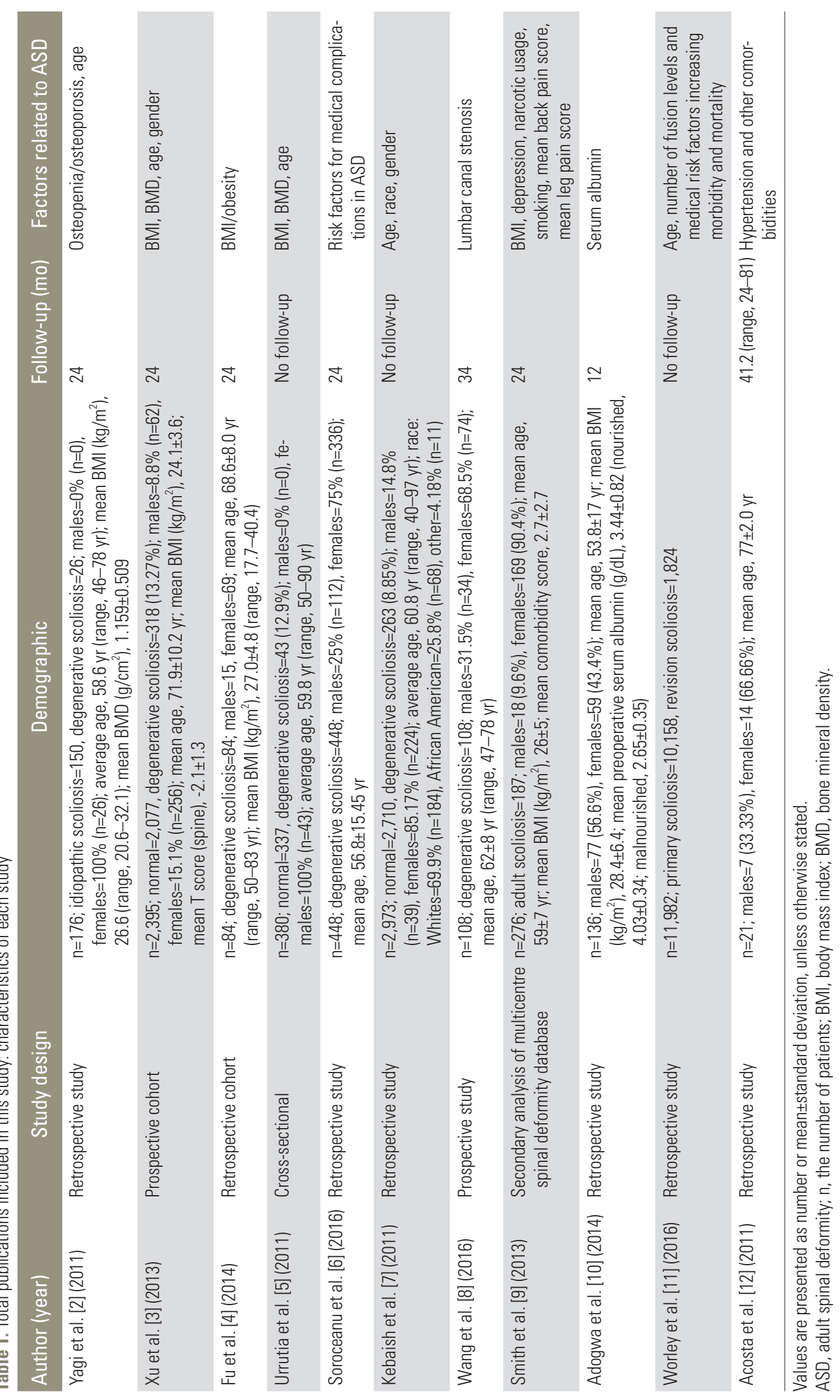




\section{Gender}

Of 11 studies included, six reported that the incidence of ASD was more common in females than that in males, with the highest range of gender difference reported by Kebaish et al. [7] $(p<0.0001)$.

\section{Age}

The average age of patients with degenerative scoliosis was $>55$ years in all studies. Kebaish et al. [7] and Xu et al. [3] subcategorized the age groups starting from 40 to $>90$ years, and the resulting prevalence rate of ASD increased as the age increased (Table 2). Worley et al. [11] reported that age $>65$ years correlated with increased morbidity and mortality compared with the 25-64 age group patients (odds ratio, 1.09).

\section{Results of body mass index}

Yagi et al. [2] in their retrospective study, which compared the BMI of ASD with the adolescent idiopathic scoliosis group, reported that the BMI of the two groups did not exhibit any statistical difference $(p=0.71)$. Likewise, the $\mathrm{BMI}$ of the scoliosis and normal groups exhibited no difference statistically $(p=0.92)$ in Xu et al. [3]. Fu et al. [4] divided cases of degenerative scoliosis into obese, overweight, and normal weight categories. Of all, 23\% were obese, and $42 \%$ were overweight. In addition, the authors reported no significant correlation between the obese groups and clinical or radiological outcomes in ASD. Conversely, Smith et al. [9] reported the BMI to be markedly related to poorest outcomes (BMI, $27 \pm 6 \mathrm{~kg} / \mathrm{m}^{2}$ ) following scoliosis surgery for adults.

\section{Results of bone mineral density}

Urrutia et al. [5] conducted a linear regression analysis to determine whether lumbar T-score, BMI, and age independently affected Cobbs angle. The age and BMI affected Cobb angle in the linear regression analysis, but lumbar T-score was not an independent predictor of Cobb angle (Table 3). Xu et al. [3] reported that lumbar T-score of the scoliosis group was significantly lower than control subjects $(-2.1 \pm 1.3$ versus $-1.7 \pm 1.6)$. Yagi et al. [2] observed only $10 \%$ osteoporosis in adult scoliosis.

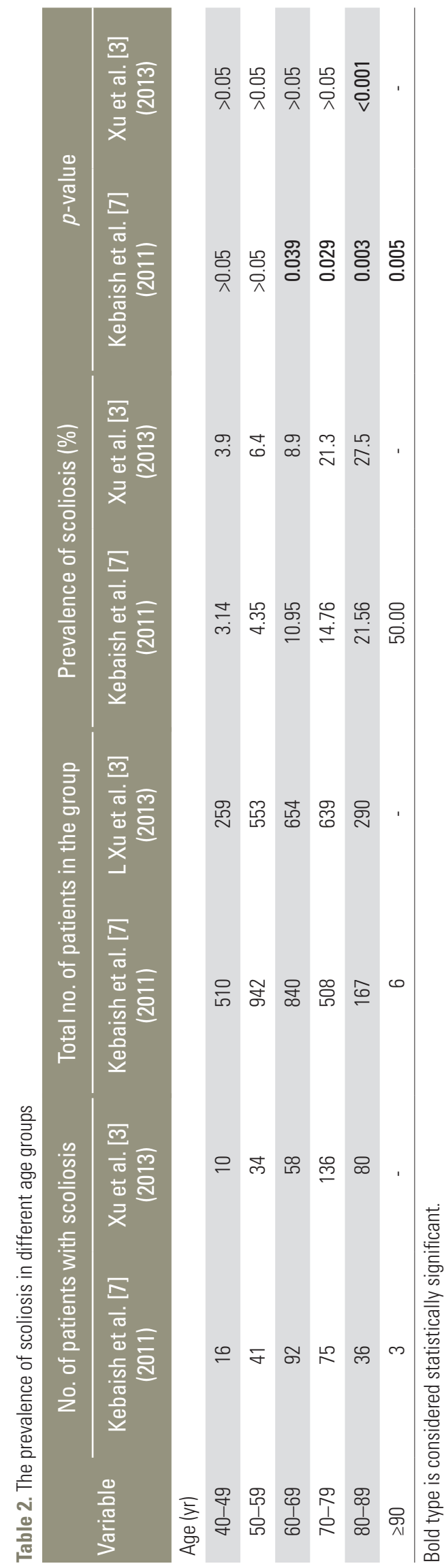


Table 3. The linear regression analysis by Urrutia et al. [5]

\begin{tabular}{lcc} 
Variable & $\beta$-coefficient & $p$-value \\
Age & 0.146 & $<0.01^{\text {a) }}$ \\
Body mass index & -0.145 & $0.06^{\text {a) }}$ \\
Lumbar T-score & 0.053 & 0.308 \\
\hline
\end{tabular}

a)Indicates significant difference.

\section{Lumbar canal stenosis}

In a series by Wang et al. [8], 32.4\% of patients with ASD are related to LCS with primary symptom being radicular pain because LCS; this group underwent lumbar decompression with or without short fusion. The results were comparable to cases in which imbalance was the primary symptom where instrumentation with long fusion was performed. Both groups did not exhibit any significant statistical difference.

\section{Other factors}

Soroceanu et al. [6] identified the risk factors causing medical complications in adult scoliosis. The univariate analysis identified the following factors significant: smoking, hypertension, heart disease, obesity, depression, duration of symptoms, the American Society of Anesthesiologists classification, and sex. After using the multivariate Poisson regression model, three independent factors were identified for the development of medical complications after ASD surgery-smoking (interest rate risk [IRR], 2.39), hypertension (IRR, 2.43), and duration of symptoms (IRR, 1.24). Smith et al. [9] reported that $38 \%$ of patients with worst outcomes following ASD surgery had a history of narcotic usage, and other $38 \%$ with worst outcomes had preoperative depression/anxiety. In a retrospective study, Acosta et al. [12] reported that only hypertension was predictive of major perioperative complication for spinal deformity surgery in patients aged $>75$ years (odds ratio, 10 ; $95 \%$ confidence interval, $1.3-78 ; p=0.02$ ).

\section{Discussion}

Classifications play a pivotal role in spine surgery as a prognostic value can be guided by effective classification systems; they are also crucial because of the insight they provide to physicians, especially in deciding the treatment protocol [13]. A classification system for spinal deformity has four main purposes-systematic categorization of similar disorders; prognosis regarding natural history and outcomes of care; correlation with health status or severity of deformity; and a guide for optimal care [14].

The management of adult scoliosis starts with analyzing clinical symptoms, related comorbid factors, and finally radiological factors. This literature review found a few of the clinical factors described below that affect the surgical outcomes of adult scoliosis.

\section{Leg pain}

In adult deformities, the clinical impact of the deformity correlates with pain and disability rather than skeletal age as in adolescent scoliosis. Claudication symptoms when walking or standing are critical symptoms of adult degenerative scoliosis. Leg pain might be caused by localized compression of root on the concave side of curve or root traction/stretch at the convex side of the curve. According to Aebi [15], short lumbosacral curves below to instrumented long thoracolumbar curves often show severe spinal stenosis at the transitional area causing leg pain.

\section{Spinal stenosis}

Patients with ASD are usually aged $>50$ years and frequently associated with stenosis [16]. There exists an active interrelation between ASD and lumbar stenosis in terms of clinical symptoms [17]. However, no universal consensus exists for surgical management of ASD related to LCS. Postacchini [18] indicated that patients whose main symptom is neurological claudication with little to no back pain and mild curve might be treated with decompression only. If back pain is the primary complaint with or without radicular symptoms, fusion could be indicated. However, Vaccaro and Ball [19] assumed that simple focal decompression alone in patients with ASD could result in further collapse, instability, and exacerbated back and leg pain.

Wang et al. [8] divided cases of ASD into the following three groups: (1) group 1: cases with primary stenosis symptom where decompression of symptomatic spinal canal stenosis and short fusion was performed; (2) group 2: cases with compensated imbalance symptom where decompression and short fusion was performed; and (3) group 3: cases with primary imbalance symptom where correction was the primary goal of management along with long fusion segments. Oswestry Disability Index 
(ODI) and Scoliosis Research Society (SRS)-22 scores markedly enhanced with the type of surgeries performed in different groups.

\section{Obesity and body mass index}

According to the World Health Organization, the BMI was categorized into four main groups as follows: obese (BMI, $\geq 30 \mathrm{~kg} / \mathrm{m}^{2}$ ); overweight (BMI, 35-29.9 $\mathrm{kg} / \mathrm{m}^{2}$ ); normal weight (BMI, 18.5-24.9 kg/m $)$ ); and underweight (BMI, $\leq 18.5 \mathrm{~kg} / \mathrm{m}^{2}$ ). To date, several studies have reported that obesity is markedly related to various health disorders $[20,21]$. In the spine, lumbar spine degeneration positively correlates with obesity [22]. Fu et al. [4] reported that surgically treated obese patients exhibited worse ODI scores than normal patients with ASD after 1-year followup $(49.1 \pm 9.0$ versus $34.8 \pm 18.4, p<0.05)$, but no significant difference was noted between all groups at 2-year followup; they concluded that obesity did not affect clinical or radiological outcomes for degenerative scoliosis after 2 years. Urrutia et al. [5] reported that age and lower BMI are independent predictors of the magnitude of the curve, that is, scoliosis was more common in older people with lower BMI. Conversely, Xu et al. [3] reported that age, gender, and BMI of Chinese population did not correlate with the severity of the curve.

\section{Osteoporosis}

Osteoporosis has been implicated in the development of degenerative scoliosis [23]. However, a few studies also suggested that degenerative scoliosis could falsely elevate spinal BMD measurements with dual-energy radiograph absorptiometry [24,25]. Yagi et al. [2] reported no correlation between curve magnitude and BMD. However, bone fusion rates were found to be lower in the osteoporosis group compared with the normal BMD group. Xu et al. [3] reported that BMD at the lumbar spine and femoral neck of scoliosis patients significantly decreased than that in the control group ( $p=0.02$ ); however, the curve severity did not correlate with BMD. Likewise, Urrutia et al. [5] reported that BMD was not an independent predictor of the magnitude of the curve.

\section{Age}

In most studies, aging increased the incidence of ASD.
Kebaish et al. [7] reported that the prevalence of scoliosis increased almost linearly from fifth to eighth decade of life. However, the curve severity did not worsen with advancing age. $\mathrm{Xu}$ et al. [3] stated that age $>65$ years could markedly contribute to the presence of adult scoliosis.

\section{Gender}

All six studies that compared the prevalence of ASD in males to females reported an increased incidence of degenerative scoliosis in females.

\section{Other factors}

According to Soroceanu et al. [6], smoking, hypertension, and duration of symptoms increase the risk of medical complications after ASD surgery. Smith et al. [9] reported that preoperative depression and narcotic usage results in worst outcomes following ASD surgery. According to Acosta et al. [12], patients aged $>75$ years with a history of hypertension are 10 times more likely to incur a major perioperative complication.

Lately, there has been a shift in the focus toward the development of the frailty index for assessing perioperative adverse events rather than chronological age alone [26-29]. To date, several methods have been tried for the development of the frailty index, and its quantification has also been confirmed [30,31]. Searle et al. [32] developed a method for creating frailty indices by using existing databases; this procedure was followed by Miller et al. [33] in creating the Adult Spinal Deformity Frailty Index using the International Spine Study Group ASD prospective patient database. Accordingly, higher patient frailty correlated with a higher risk of complications, including pseudoarthrosis, proximal junctional kyphosis, wound infection, and longer hospital stay.

\section{Review of existing classifications}

The initial classification system for scoliosis by the Terminology Committee of the SRS in 1969 was basically etiological without considering the curve patterns and its location [34]. Ponseti and Friedman [35] developed a classification based on the curve location but it had limitations regarding guidance to the treatment. Later, several other classifications were described, but all primarily concentrated on adolescent deformity [36-38]. To date, 
extensive research has been conducted on ASD classification in the last decade especially by Frank J. Schwab and SRS $[34,39]$.

Aebi [15] proposed a classification based on the etiology of the curve, which was designed primarily to correlate with a natural history of the deformity but could not indicate surgical management of ASD. Simmons [40] classified adult scoliosis into two groups based on the presence or absence of the vertebral rotation.

Schwab et al. [41] proposed a classification of adult scoliosis, which includes a simple system of graded clinical impact parameters (apex, lumbar lordosis, and intervertebral subluxation). The inter- and intraobserver reliability of the classification has been reported excellent. Furthermore, reported surgical outcomes closely correlated with the described radiographic modifiers. However, the classification system could not arrive at treatment algorithms and surgical planning for adults with spinal deformity.

SRS classification included major curve types, sagittal modifiers, and global balance modifiers, which guided optimal care in management of adult scoliosis [34]; however, this classification did not encompass clinical considerations such as patients' symptoms and related comorbidities.

SRS in association with Schwab et al. [41] developed a classification which contains four components as follows: curve type; pelvic incidence minus lumbar lordosis modifier; pelvic tilt modifier; and global balance modifier. The advantage of this classification is that it considers spinopelvic parameters, which were not considered previously. All the modifiers strongly correlated with health-related quality of life. Hence, this classification acted as a guideline for optimizing surgical planning for ASD. The validity and reliability of the classification was performed by Liu et al. [42], who stated that SRS-Schwab classification system was simple and comprehensive with sufficient reproducibility. However, the limitation of the classification is that it does not considers coexisting clinical conditions, which probably plays a pivotal role in elderly patients.

This review revealed the significance of neurological symptoms, spinal stenosis, osteoporosis, obesity, age, and smoking, which markedly affect the management of ASD, and none of these are addressed in any of the present classification systems (Table 4). With the increasing number of patients being diagnosed and treated with ASD, it is imperative to comprehensively classify these patients into clinicoradiological subgroups.

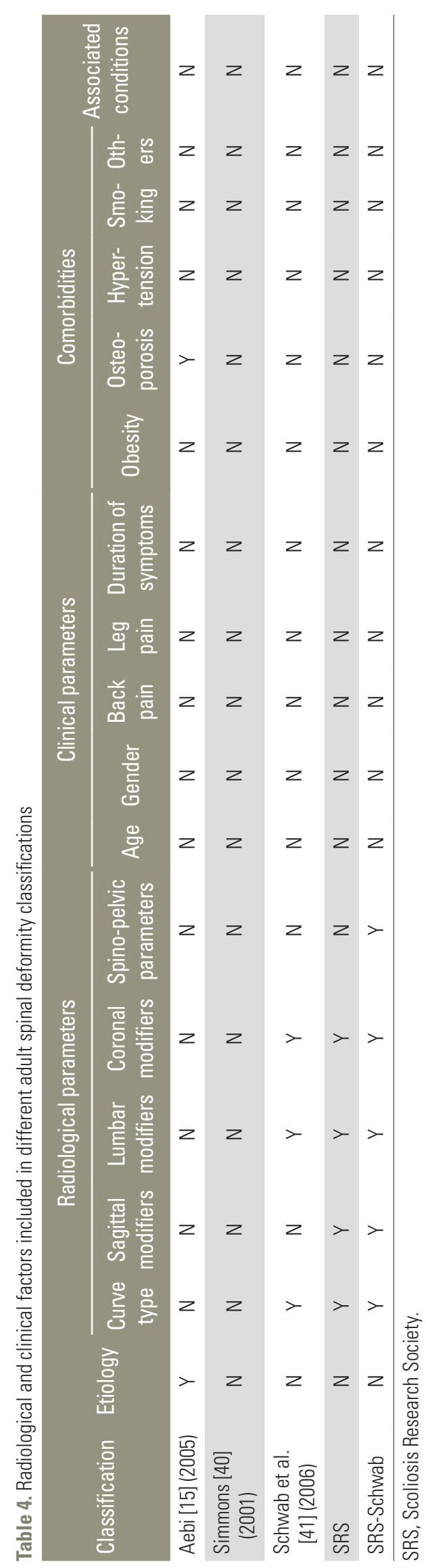




\section{Conclusions}

Classifying patients with ASD based on radiological parameters will not address the full spectrum of the ASD pathology. Although radiologically similar, patients with coexisting neurological symptoms, pain, and comorbidities are completely different in terms of disability and treatment. An ideal classification system should necessarily suggest treatment options for the disorder. None of the available classification systems address all these factors. Hence, there is an urgent need for a comprehensive classification system for ASD, which not only classifies patients but also suggests treatment options.

\section{Conflict of Interest}

No potential conflict of interest relevant to this article was reported.

\section{Acknowledgments}

The authors acknowledge the guidance and cooperation of AO Spine Knowledge Forum (Deformity) and AO Spine International.

\section{ORCID}

Arun-Kumar Viswanadha: https://orcid.org/0000-00026322-4357

\section{Author Contributions}

Conception and design, supervision: JNB; data acquisition, analysis of data, drafting of the manuscript: AKV; and critical revision: JNB, MI, JBP.

\section{References}

1. Kostuik JP. Adult scoliosis: the lumbar spine. In: Bridwell KH, DeWald RL, Hammerberg KW, editors. The textbook of spinal surgery. 2nd ed. Philadelphia (PA): Lippincott-Raven; 1997. p. 733-75.

2. Yagi $M$, King $A B$, Boachie-Adjei O. Characterization of osteopenia/osteoporosis in adult scoliosis: does bone density affect surgical outcome? Spine (Phila Pa 1976) 2011;36:1652-7.

3. Xu L, Sun X, Huang S, et al. Degenerative lumbar scoliosis in Chinese Han population: prevalence and relationship to age, gender, bone mineral density, and body mass index. Eur Spine J 2013;22:1326-31.

4. Fu L, Chang MS, Crandall DG, Revella J. Does obesity affect surgical outcomes in degenerative scoliosis? Spine (Phila Pa 1976) 2014;39:2049-55.

5. Urrutia J, Diaz-Ledezma C, Espinosa J, Berven SH. Lumbar scoliosis in postmenopausal women: prevalence and relationship with bone density, age, and body mass index. Spine (Phila Pa 1976) 2011;36:73740.

6. Soroceanu A, Burton DC, Oren JH, et al. Medical complications after adult spinal deformity surgery: incidence, risk factors, and clinical impact. Spine (Phila Pa 1976) 2016;41:1718-23.

7. Kebaish KM, Neubauer PR, Voros GD, Khoshnevisan MA, Skolasky RL. Scoliosis in adults aged forty years and older: prevalence and relationship to age, race, and gender. Spine (Phila Pa 1976) 2011;36:731-6.

8. Wang G, Cui X, Jiang Z, Li T, Liu X, Sun J. Evaluation and surgical management of adult degenerative scoliosis associated with lumbar stenosis. Medicine (Baltimore) 2016;95:e3394.

9. Smith JS, Shaffrey CI, Glassman SD, et al. Clinical and radiographic parameters that distinguish between the best and worst outcomes of scoliosis surgery for adults. Eur Spine J 2013;22:402-10.

10. Adogwa O, Martin JR, Huang K, et al. Preoperative serum albumin level as a predictor of postoperative complication after spine fusion. Spine (Phila Pa 1976) 2014;39:1513-9.

11. Worley N, Marascalchi B, Jalai CM, et al. Predictors of inpatient morbidity and mortality in adult spinal deformity surgery. Eur Spine J 2016;25:819-27.

12. Acosta FL Jr, McClendon J Jr, O'Shaughnessy BA, et al. Morbidity and mortality after spinal deformity surgery in patients 75 years and older: complications and predictive factors. J Neurosurg Spine 2011;15:667-74.

13. Garbuz DS, Masri BA, Esdaile J, Duncan CP. Classification systems in orthopaedics. J Am Acad Orthop Surg 2002;10:290-7.

14. Audige L, Bhandari M, Hanson B, Kellam J. A concept for the validation of fracture classifications. J Orthop Trauma 2005;19:401-6.

15. Aebi M. The adult scoliosis. Eur Spine J 2005;14:92548. 
16. Robin GC, Span Y, Steinberg R, Makin M, Menczel J. Scoliosis in the elderly: a follow-up study. Spine (Phila Pa 1976) 1982;7:355-9.

17. Marty-Poumarat C, Scattin L, Marpeau M, Garreau de Loubresse C, Aegerter P. Natural history of progressive adult scoliosis. Spine (Phila Pa 1976) 2007;32:1227-34.

18. Postacchini F. Surgical management of lumbar spinal stenosis. Spine (Phila Pa 1976) 1999;24:1043-7.

19. Vaccaro AR, Ball ST. Indications for instrumentation in degenerative lumbar spinal disorders. Orthopedics 2000;23:260-71.

20. Hensrud DD, Klein S. Extreme obesity: a new medical crisis in the United States. Mayo Clin Proc 2006;81(10 Suppl):S5-10.

21. Hangai M, Kaneoka K, Kuno S, et al. Factors associated with lumbar intervertebral disc degeneration in the elderly. Spine J 2008;8:732-40.

22. Carter OD, Haynes SG. Prevalence rates for scoliosis in US adults: results from the first National Health and Nutrition Examination Survey. Int J Epidemiol 1987;16:537-44.

23. Girardi FP, Parvataneni HK, Sandhu HS, et al. Correlation between vertebral body rotation and twodimensional vertebral bone density measurement. Osteoporos Int 2001;12:738-40.

24. Pappou IP, Girardi FP, Sandhu HS, et al. Discordantly high spinal bone mineral density values in patients with adult lumbar scoliosis. Spine (Phila Pa 1976) 2006;31:1614-20.

25. Rand T, Seidl G, Kainberger F, et al. Impact of spinal degenerative changes on the evaluation of bone mineral density with dual energy X-ray absorptiometry (DXA). Calcif Tissue Int 1997;60:430-3.

26. Farhat JS, Velanovich V, Falvo AJ, et al. Are the frail destined to fail? Frailty index as predictor of surgical morbidity and mortality in the elderly. J Trauma Acute Care Surg 2012;72:1526-30.

27. Joseph B, Pandit V, Sadoun M, et al. Frailty in surgery. J Trauma Acute Care Surg 2014;76:1151-6.

28. Kim SW, Han HS, Jung HW, et al. Multidimensional frailty score for the prediction of postoperative mortality risk. JAMA Surg 2014;149:633-40.

29. Schuurmans H, Steverink N, Lindenberg S, Frieswijk N, Slaets JP. Old or frail: what tells us more? J Geron- tol A Biol Sci Med Sci 2004;59:M962-5.

30. Rockwood K, Andrew M, Mitnitski A. A comparison of two approaches to measuring frailty in elderly people. J Gerontol A Biol Sci Med Sci 2007;62:73843.

31. Rockwood K, Song X, MacKnight C, et al. A global clinical measure of fitness and frailty in elderly people. CMAJ 2005;173:489-95.

32. Searle SD, Mitnitski A, Gahbauer EA, Gill TM, Rockwood K. A standard procedure for creating a frailty index. BMC Geriatr 2008;8:24.

33. Miller EK, Neuman BJ, Jain A, et al. An assessment of frailty as a tool for risk stratification in adult spinal deformity surgery. Neurosurg Focus 2017;43:E3.

34. Lowe T, Berven SH, Schwab FJ, Bridwell KH. The SRS classification for adult spinal deformity: building on the King/Moe and Lenke classification systems. Spine 2006;31(19S):S119-S25.

35. Ponseti IV, Friedman B. Prognosis in idiopathic scoliosis. J Bone Joint Surg Am 1950;32A:381-95.

36. King HA, Moe JH, Bradford DS, Winter RB. The selection of fusion levels in thoracic idiopathic scoliosis. J Bone Joint Surg Am 1983;65:1302-13.

37. Lenke LG, Betz RR, Clements D, et al. Curve prevalence of a new classification of operative adolescent idiopathic scoliosis: does classification correlate with treatment? Spine (Phila Pa 1976) 2002;27:604-11.

38. Lenke LG, Betz RR, Harms J, et al. Adolescent idiopathic scoliosis: a new classification to determine extent of spinal arthrodesis. J Bone Joint Surg Am 2001;83-A:1169-81.

39. Schwab F, Ungar B, Blondel B, et al. Scoliosis Research Society-Schwab adult spinal deformity classification: a validation study. Spine (Phila Pa 1976) 2012;37:1077-82.

40. Simmons ED. Surgical treatment of patients with lumbar spinal stenosis with associated scoliosis. Clin Orthop Relat Res 2001;(384):45-53.

41. Schwab F, Farcy JP, Bridwell K, et al. A clinical impact classification of scoliosis in the adult. Spine (Phila Pa 1976) 2006;31:2109-14.

42. Liu Y, Liu Z, Zhu F, et al. Validation and reliability analysis of the new SRS-Schwab classification for adult spinal deformity. Spine (Phila Pa 1976) 2013;38:902-8. 